\title{
Fluorescence-Encoded Gold Nanoparticles: Library Design and Modulation of Cellular Uptake into Dendritic Cells
}

\author{
Laura Rodriguez-Lorenzo, * Kleanthis Fytianos, Fabian Blank, \\ Christophe von Garnier, Barbara Rothen-Rutishauser, and Alke Petri-Fink*
}

\begin{abstract}
In order to harness the unique properties of nanoparticles for novel clinical applications and to modulate their uptake into specific immune cells we designed a new library of homo- and hetero-functional fluorescence-encoded gold nanoparticles (Au-NPs) using different poly(vinyl alcohol) and poly(ethylene glycol)-based polymers for particle coating and stabilization. The encoded particles were fully characterized by $U V$-Vis and fluorescence spectroscopy, zeta potential and dynamic light scattering. The uptake by human monocyte derived dendritic cells in vitro was studied by confocal laser scanning microscopy and quantified by fluorescenceactivated cell sorting and inductively coupled plasma atomic emission spectroscopy. We show how the chemical modification of particle surfaces, for instance by attaching fluorescent dyes, can conceal fundamental particle properties and modulate cellular uptake. In order to mask the influence of fluorescent dyes on cellular uptake while still exploiting its fluorescence for detection, we have created hetero-functionalized $A u-N P s$, which again show typical particle dependent cellular interactions. Our study clearly prove that the thorough characterization of nanoparticles at each modification step in the engineering process is absolutely essential and that it can be necessary to make substantial adjustments of the particles in order to obtain reliable cellular uptake data, which truly reflects particle properties.
\end{abstract}

Dr. L. Rodriguez-Lorenzo, K. Fytianos,

Prof. B. Rothen-Rutishauser, Prof. A. Petri-Fink

Adolphe Merkle Institute

University of Fribourg

Route de l'Ancienne Papeterie

P.O. Box 209, Marly 1723 (Switzerland)

E-mail: laura.rodriguez-lorenzo@unifr.ch;

alke.fink@unifr.ch

Prof. A. Petri-Fink

Department of Chemistry

University of Fribourg

Chemin du Musee 9

Fribourg 1700 (Switzerland)

Dr. F. Blank, Dr. C. von Garnier, Prof. B. Rothen-Rutishauser

Respiratory Medicine

Bern University Hospital,

Inselspital, Freiburgstrasse, Bern 3010 (Switzerland)

\section{Introduction}

There is a strong requirement to understand the biological behavior of living organisms at the molecular level and this places a significant demand on current high-throughput analysis and imaging systems. ${ }^{[1,2]}$ The utilization of so-called encoded particles for the detection or targeting of specific cells or intracellular compartments is one of the most powerful tools. Several examples of encoded particles have been described based on different parameters such as composition, size, morphology, lithography barcodes or spectroscopic properties (e.g., electronic or vibrational profiles). The codification of particles through highly active fluorescence reporters is also a promising method. ${ }^{[2-7]}$ Fluorescence generates very intense signals that allow detection in very short data acquisition times with straightforward analysis. ${ }^{[2]}$ In fact, fluorescence-encoded particles can be rapidly processed by using methods that detect fluorescence in single 
cells or tissues such as confocal laser scanning microscopy (LSM), fluorescence-activated cell-sorting (FACS) ${ }^{\left[{ }^{[5-8]}\right.}$ or by advanced imaging techniques to detect fluorescence in small animals. ${ }^{[9,10]}$ Thus, such fluorescently encoded particles offer a sensitive, rapid and cost-effective solution for diagnostic and imaging applications.

Gold nanoparticles (Au-NPs) are the key element in the synthesis of highly active encoded particles, because of their unique scattering and absorption properties arising from localized surface plasmon resonances (LSPRs), ${ }^{[11]}$ as well as offering a wide number of well-controlled synthetic approaches. ${ }^{[12]}$ Specifically, chemical functionalization of the Au-NP surface is usually performed to add biocompatibility and specificity, ${ }^{[12]}$ a large variety of polymers are currently used to modify the Au surface. The shell can render the particle biocompatible, and also protects NPs for example against oxidation, reduces aggregation and allows for the attachment of targeting ligands (e.g., antibodies) and labels (e.g., fluorophores). ${ }^{[5,13,14]}$

We have developed a novel design to establish a simple and reproducible library of fluorescence-encoded Au-NPs with different surface properties. We have selected functionalized polyethylene glycol (PEG) and polyvinyl alcohol (PVA) polymer probes for Au-NP coating to build up the encoded NP library, since it was shown in previous studies that those polymers improve the colloidal stability and biocompatibility of NPs in vivo and in vitro. ${ }^{[5,8,15,16]}$ In addition, their chemical difference (e.g., degree of hydrophobicity) allows investigation of these properties since it is well-known that the functional groups of the polymer can modify the Au-NPs characteristics, modulating their self-assembly or allowing selective interactions. ${ }^{[17,18]}$ Quantitative cell uptake in vitro studies have already demonstrated that size, shape, coating and surface charge can modulate the internalization of NPs (e.g., Au, QDs, silica). ${ }^{[18,19]}$ Many studies, in serumfree and serum-containing media, have reported that cells preferentially incorporate positively charged NPs rather than negatively charged ones. ${ }^{[13]}$ Furthermore, cytotoxicity assays have often revealed increased cytotoxic responses of positively charged NPs. In addition, many studies have shown a size-dependency of NP internalization and cytotoxic behavior. ${ }^{[20,21]}$

In particular $\mathrm{Au}$ or silica NPs have been investigated in many in vitro studies and the impact of surface charge and surface adsorbed proteins, i.e. the so-called protein corona, on cellular uptake was studied extensively for those systems. ${ }^{[22]}$ Other factors such as shape have gained more interest recently. Hutter et al. ${ }^{[23]}$ for example have compared the interactions of microglia and neurons with $\mathrm{Au}$ spheres, rods, and urchins while Kohlar et al.[24] have even exploited the shape differences to target antibody-coated NPs to lung and brain endothelium. In another study it was shown that nanohexapods exhibited the highest cellular uptake and lowest cytotoxicity compared to nanorods and nanocages in-vitro. ${ }^{[25]}$ However, the influence of particle morphology on the particle-cell interaction is not yet completely understood. ${ }^{[24]}$ Particle shape, such like particle charge, is usually not independent of other parameters such as surface chemistry and unraveling the influence of shape on NP-cell interactions from other generally convoluted variables is a challenging problem. ${ }^{[18]}$

Notably, the impact of seemingly minor surface modifications, such as the attachment of fluorescent dyes, on cellular responses has not as yet been studied in detail. Since fluorescent probes are abundantly attached to NPs to facilitate their detection and quantification in in vitro and in vivo studies, it is absolutely crucial to understand the effect of those small molecules on particle properties and subsequent biological interactions.

In this study, we show how the presence of fluorescent dyes can alter particle internalization in human monocytederived dendritic cells (MDDC) in vitro. As a consequence, we have developed and tested an optimized fluorescenceencoded Au-NPs library that allows for cell uptake studies, which are not falsified by the presence of surface molecules.

Our study highlights the importance of a comprehensive interdisciplinary approach to design tailor-made encoded NPs with different surface functionalization to understand their complex behavior in biological fluids such as cell culture media and to study the interaction on single cell level by cutting-edge techniques before further biomedical applications can be developed.

\section{Results and Discussion}

Figure 1 schematically describes the encoded NP library. The detailed synthetic approaches are described in the Experimental Section. Briefly, the selected fluorophore was bound to six different polymers following standard bioconjugation techniques, resulting in highly dye functionalized polymers (Figure 2a and Figure S1). Thereafter, six batches of monodisperse $15 \mathrm{~nm}$ Au-NPs (Figure 2b) were coated with those six dye-conjugated polymers. PEG layers were covalently bound to Au-NPs through the thiol functional group, ${ }^{[26]}$ while coating with PVA was achieved through electrostatic interaction between the Au surface and the polymer. In order to examine the influence of the surface charge on MDDC uptake, amine-functional (e.g., amine-PEG) and carboxylicfunctional PEG and PVA were respectively used. Au-NPs were also coated using a mixture of PEG and PVA, providing the possibility to investigate the influence of the "extra" polymer on the particle on cellular interactions. This set of NPs is referred to as "homo-functional encoded Au-NPs" and displayed the fluorophore on the particle surfaces (Figure 1a).

However, since quenching of fluorophores by Au-NPs is a well-described phenomenon, ${ }^{[27]}$ we also designed a second set of particles, herein referred to as "hetero-functional encoded Au-NPs", where the fluorophore was shielded by a second polymer coating by capping two alternating monolayers on NP surface. Thus, as an example, Au-NPs were initially coated with dye-conjugated amine-PEG followed by the addition of the analogous unlabeled polymer. In this set-up, the fluorophore was now "sandwiched" between 2 polymer layers (Figure 1b). The localization of fluorophores with respect to the gold surface was controlled by utilizing 


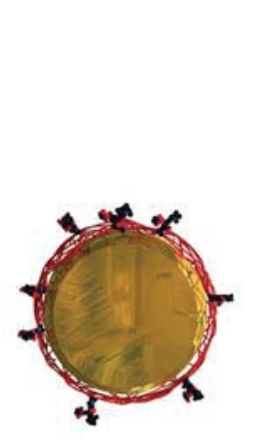

(a) Set of Homo-functional encoded Au NPs

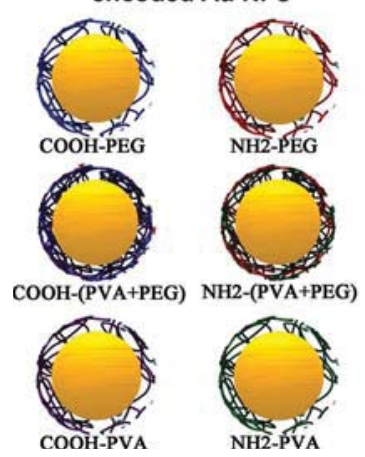

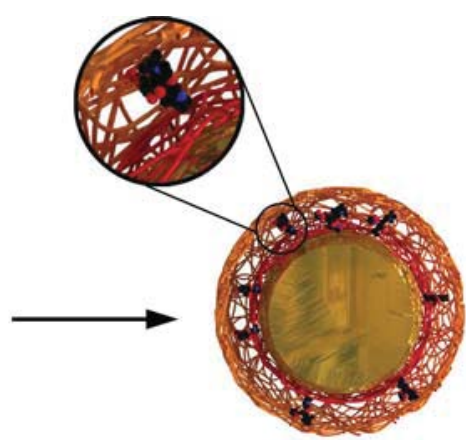

(b) Set of Hetero-functional encoded Au NPs

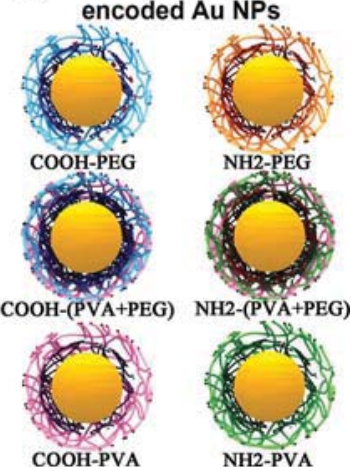

Figure 1. Schematic illustration of the designed fluorescence encoded Au-NPs. (a) The homo-functional encoded NPs consist of a core of Au-NPs coated with a fluorophore-conjugated polymer: amine- or carboxylic- functional thiolated PEG (homo-NH2-PEG and homoCOOH-PEG, respectively), PVA (homo-NH2-PVA and homo-COOH-PVA, respectively) or a mixture of both (homo-NH2-(PVA+PEG) and homo$\mathrm{COOH}-(\mathrm{PVA}+\mathrm{PEG})$, respectively). (b) The second set of encoded particles presents a two-layer coating of the Au core with a fluorophore-conjugated polymer covered by an unlabeled polymer. The hetero-functional set is composed of six particles: amine- and carboxylic functional thiolated PEG (hetero-NH2-PEG and hetero-COOH-PEG, respectively), PVA (hetero$\mathrm{NH} 2$-PVA and hetero-COOH-PVA, respectively) or the mixture of both (hetero-NH2-(PVA+PEG) and hetero-COOH-(PVA+PEG), respectively). Zoom: Illustration of fluorescent dye "sandwiched" between the two polymer layers. The chemical structures of both particle sets are depicted in more detail in Figure S1 in the Supporting information.

appropriate spacers, which allow compensation for fluorescence quenching effects of Au-NPs. ${ }^{[4,9,10]}$ It has been shown before that if the separation of the fluorophore from the $\mathrm{Au}$ surface is insufficient (below $6 \mathrm{~nm}$ ), ${ }^{[28]}$ a loss of the fluorescence signal is observed. ${ }^{[29]}$ In fact, the previously reported low fluorescence intensities for polymer-coated Au-NPs are most likely due to the diffusion of the dye molecule or inadequate distances to the gold surface. ${ }^{[30]}$

We overcame these challenges by (i) covalent attachment of the fluorophore to the polymers and (ii) using high molecular-weight polymers. Fluorescence from both encoded particles sets was measured according to their excitation and emission spectra (Figure S2), demonstrating a high fluorescence intensity for both sets and thus confirming the successful spacer function of the polymer layer (Figure S3). However, the number of fluorophore molecules per Au-NPs showed significant differences depending on the polymer that was employed (Figure 2a). PEG contains only one terminal functional group, while PVA has a large number of functional

groups available (Figure S1). All NPs were stable and showed no evidence for aggregation, red shift or band broadening in the UV-Vis spectra (Figures 2c-d). The hydrodynamic diameters and zeta potentials for all encoded and unlabeled particles are summarized in Table 1. Analysis by dynamic light scattering (DLS) showed that homo-functional encoded Au-NPs presented similar hydrodynamic diameters to their analogue unlabeled particles, but the average diameters of hetero-functional encoded particles were in general higher than the homo-functional particles.

Interestingly, unlabeled carboxyl-functional PEG Au-NPs displayed a larger hydrodynamic diameter than their dyeencoded homo-functional analogues. This was already described by Manson et al. ${ }^{[31]}$ who showed that the hydrodynamic diameter of Au-NPs increased from $8 \mathrm{~nm}$ to $19 \mathrm{~nm}$ as a function of capping density. Based on this, we created a hypothesis that the observed difference between the diameters of unlabeled and homo-COOH-PEG could be due to different PEG surface coverage. Also, hetero-NH2-PVA and hetero-NH2-(PVA+PEG) showed smaller diameters compared to the homo-functional counterparts (Table 1). This inverse tendency was probably caused by electrostatic interactions. Charged polymers exhibit pH-dependent swelling behavior. As the degree of ionization increases, the number of fixed charges also increase, resulting in stronger electrostatic repulsions between the chains. ${ }^{[32,33]}$ However, electrostatic attraction is favored at $\mathrm{pH}$ values where both functional groups are charged when usually multilayers are constructed by the consecutive alternate buildup of oppositely charged polymers. Abe et al. ${ }^{[34]}$ reported that poly(vinyl pyrrolidone)/ poly(methacrylic acid) capsules were stable up to $\mathrm{pH} 7$ where they showed their lowest diameter. Similar results were reported for flat multilayers of 10 layers of poly(vinyl pyrrolidone)/ poly(acrylic acid) by Cho and Caruso. ${ }^{[35]}$ Zeta potential measurements (Table 1) revealed negatively charged surfaces for all, i.e. unlabeled as well as homo- and hetero functional, carboxylic functional(-COO $\left.{ }^{-}\right) \mathrm{Au}-\mathrm{NPs}$. Interestingly, although unlabeled NH2-PEG and NH2-(PVA+PEG) Au-NPs showed positively charged amine groups $\left(-\mathrm{NH}_{3}{ }^{+}\right)$ on the surface, their zeta potential remained negative, which might be attributed to the reduced number of protonated amino groups (1.5-2 fold; $\mathrm{pKa} \sim 8.5-9.5)$ at neutral $\mathrm{pH}$ and the strongly negative zeta potential of original citrate Au-NPs $(-39.5 \mathrm{mV}$ at $\mathrm{pH} 7)$. This was previously reported also for PEGylated quantum dots. ${ }^{[36]}$ Interestingly, all homo-functional particles showed very similar negative zeta potentials $(-16$ to $-20 \mathrm{mV})$. We attribute this to the conjugation of the particles to the fluorophore, which changed the zeta potential because of the conversion of the deprotonated carboxylic $\left(-\mathrm{COO}^{-}, \mathrm{pKa} \sim 2-3.5\right)$ and protonated surface amine $\left(\mathrm{NH}_{3}{ }^{+}\right)$ groups to amides. ${ }^{[37]}$ In contrast, the hetero-encoded particles displayed the expected positive zeta potentials for the aminefunctional layers and negative charges for the carboxylicfunctional layers.

It is well known that Au-NPs can aggregate in solutions containing proteins or high salt concentrations, such as cell culture media supplemented with fetal calf serum (FCS), and that the formation of NP aggregates can influence their behavior in cell culture media and therefore cell uptake. ${ }^{[38]}$ 


\begin{tabular}{lc} 
(a) $\begin{array}{c}\text { Fluorescence } \\
\text { encoded Au NPs }\end{array}$ & $\begin{array}{c}\text { Fluorophore molecules per Au } \\
\text { NP }( \pm \text { SD) }\end{array}$ \\
\hline NH2-PEG & $369(50)$ \\
NH2-(PVA+PEG) & $1050(113)$ \\
NH2-PVA & $3410(132)$ \\
COOH-PEG & $450(26)$ \\
COOH(PVA+PEG) & $801(74)$ \\
COOH-PVA & $314(78)$ \\
\hline
\end{tabular}
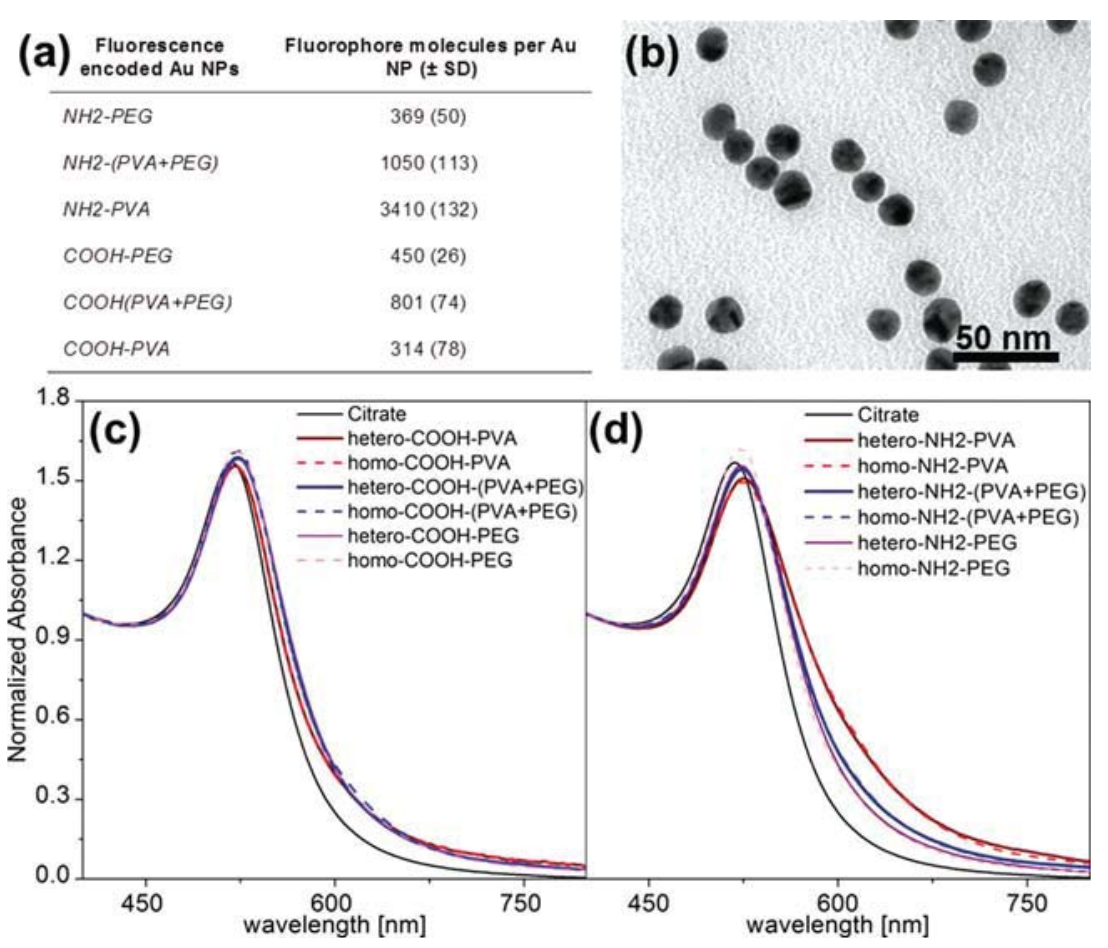

Figure 2. (a) Number of dye (ATTO 590 or AF 594) molecules per Au-NP. (b) TEM image of $15 \mathrm{~nm}$-citrate-coated Au-NPs used as core in the synthesis of the encoded particle library. UV-Vis spectra of homo- and hetero-functional encoded Au-NPs. Polymers: (c) carboxylfunctional polymers (PVA, PEG or PVA+PEG mixture) and (d) amine-functional polymers (PVA, PEG or PVA+PEG mixture).

This aggregation phenomenon produces a red shift of the LSPR to longer wavelengths visible by UV-Vis spectroscopy. ${ }^{[11]}$ We tested the colloidal stability of all encoded particles in RPMI 1640 medium with 10\% FCS at cell incubation conditions $\left(37^{\circ} \mathrm{C}\right.$ and $\left.5 \% \mathrm{CO}_{2}\right)$ after 1 and $24 \mathrm{~h}$. None of the particles aggregated in complete cell culture medium at any time (Figure S4).

The cellular internalization of all Au-NPs was studied using MDDCs, which are key antigen-presenting cells orchestrating both innate and adaptive immune functions. ${ }^{[39]}$ No morphological changes were observed in phase contrast images of living cells (Figure S6) as well as in LSM images of fixed cells labeled for the cell nuclei and F-Actin. In addition to assessing cell morphology, cytotoxicity was measured by the lactate dehydrogenase (LDH) assay (Figure S7). As higher concentrations of Au-NPs have been shown to interfere with colorimetric assays such as the LDH assay (data not presented), cytotoxicity was additionally measured by the Trypan blue exclusion method (Figure S8), which confirmed the LDH results. The quantification of particle internalization or particle-cell surface interaction, respectively, was investigated by Inductively Coupled Plasma Atomic Emission Spectroscopy (ICPOES) to measure the Au content.

Figure 3a summarizes the particle uptake of the three particle series (i.e., unlabeled polymer coated ("unlabeled homo-functionalized"), dye-labeled monolayer polymer coated ("homo-functionalized), and dye-sandwiched polymer coated ("hetero-functionalized) Au-NPs for all 6 different polymer types. Figures $3 b-d$ describe the particle uptake by MDDCs as a function of the different parameters studied, i.e., presence of fluorescent dye, polymer type, and surface charge.

The unlabeled and hetero-functional encoded Au-NPs displayed a similar trend of particle internalization, whereas all homo-functional encoded particles exhibited comparably low uptake in MDDCs. Figure 3a-d clearly show that the internalization of homo-functional encoded particles does not depend on the type of polymer or the surface functional group of the polymer backbone. We therefore conclude that the internalization of encoded homo-functionalized particles depended, at least in part, on the presence of the fluorophore at the NP surface, which seems to fully mask the underneath polymer (type and chemical functional group).

The importance of the surface on particle endocytosis by cells has already been well described. ${ }^{[18,19]}$ Thus, comparable

Table 1. Size and zeta potential of polymer coated Au-NPs.

\begin{tabular}{|c|c|c|c|c|c|c|}
\hline & \multicolumn{2}{|c|}{ Unlabeled Homo-functionalized } & \multicolumn{2}{|c|}{ Homo-functionalized } & \multicolumn{2}{|c|}{ Hetero-functionalized } \\
\hline & $\begin{array}{c}\text { Size }^{\mathrm{a})}\left(P D^{\mathrm{b}}\right) \\
{[\mathrm{nm}]}\end{array}$ & $\begin{array}{l}\text { Zeta-potential }{ }^{\mathrm{c})} \\
{[\mathrm{mV}]}\end{array}$ & $\begin{array}{c}\operatorname{Size}^{\mathrm{a})}\left(\mathrm{PD}^{\mathrm{b})}\right) \\
{[\mathrm{nm}]}\end{array}$ & $\begin{array}{l}\text { Zeta-potentialc) } \\
{[\mathrm{mV}]}\end{array}$ & $\begin{array}{c}\operatorname{Size}^{\mathrm{a})}\left(\mathrm{PD}^{\mathrm{b})}\right) \\
{[\mathrm{nm}]}\end{array}$ & $\begin{array}{c}\text { Zeta-potential }^{\text {c) }} \\
{[\mathrm{mV}]}\end{array}$ \\
\hline NH2-PEG & $19.5(9.0)$ & $-21.9 \pm 8.2$ & $18.4(9.1)^{\star}$ & $-20.1 \pm 6.6$ & $32.3(14.6)^{\star \star}$ & $20.4 \pm 7.1$ \\
\hline$N H 2-(P V A+P E G)$ & $53.9(19.8)$ & $-24.1 \pm 4.9$ & $53.5(20.0)$ & $-20.3 \pm 6.2$ & $33.5(14.6)^{\star \star}$ & $22.1 \pm 5.7$ \\
\hline NH2-PVA & 71.9 (31.9) & $7.6 \pm 2.8$ & $64.1(33.0)^{\star}$ & $-18.7 \pm 5.7$ & $50.0(22.6)^{\star \star}$ & $17.0 \pm 6.8$ \\
\hline $\mathrm{COOH}-\mathrm{PEG}$ & $34.5(15.3)$ & $-19.9 \pm 6.5$ & $19.1(7.1)^{\star}$ & $-16.5 \pm 3.5$ & $23.9(10.6)^{\star \star}$ & $-36.6 \pm 13.2$ \\
\hline $\mathrm{COOH}-(\mathrm{PVA}+\mathrm{PEG})$ & $22.1(6.1)$ & $-32.3 \pm 6.2$ & $22.4(6.3)$ & $-18.5 \pm 8.2$ & $32.2(15.1)^{\star \star}$ & $-24.5 \pm 9.2$ \\
\hline $\mathrm{COOH}-\mathrm{PVA}$ & $21.1(9.0)$ & $-34.4 \pm 13.6$ & $21.1(10.6)$ & $-17.4 \pm 3.7$ & $28.5(13.1)^{\star \star}$ & $-22.4 \pm 7.1$ \\
\hline
\end{tabular}

a) Mean hydrodynamic diameter obtained by dynamic light scattering (DLS) at room temperature and at a scattering angle of $90^{\circ}$ (Figure S5 and Table S1). DLS measurements were carried out in triplicate; ${ }^{b}$ ) Polydispersity (\%); ${ }^{c}$ Zeta-potentials were measured in 10 runs (mean $\pm \mathrm{SD}$ ). ${ }^{*}:{ }^{*} p<0.05$ (pairwise t-test): unlabeled versus homo-functionalized Au-NPs. ${ }^{*}$ : ${ }^{*} p<0.05$ (pairwise t-test): homo- versus hetero-functionalized Au-NPs. 


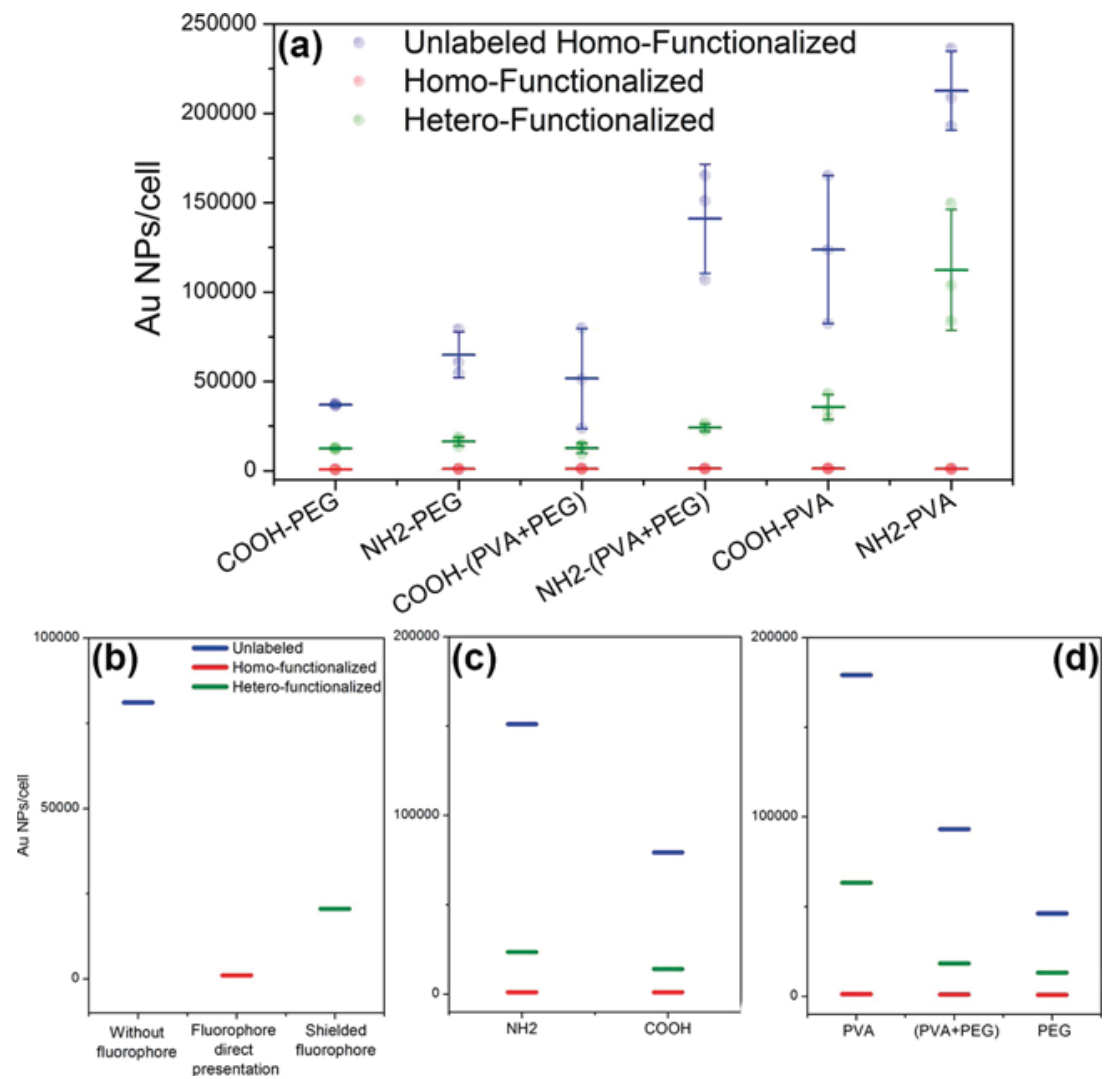

Figure 3. (a) Quantification of intracellular Au by ICP-OES shows the influence of cellular uptake as a function of the surface modification of unlabeled homo-functionalized Au-NPs (blue), Homo-functionalized Au-NPs (red), and Hetero-functionalized Au-NPs (green). MDDCs were incubated with $20 \mu \mathrm{gmL}^{-1} \mathrm{NPs}$ for $15 \mathrm{~h}$. Samples were measured in triplicate (light red, green and blue circles). The horizontal lines indicate the mean. (b-d) Parameters that modulate cellular uptake: (b) Presence of fluorophore; (c) Functional group and (d) Type of polymer. The horizontal lines indicate the median.

cellular uptake could be explained by the very similar negative zeta-potentials of all homo-functionalized particles independent of the polymer used (Table 1). Dendritic cells (DCs) are antigen presenting cells that take up antigens by several mechanisms such as receptor-mediated endocytosis, macropinocytosis and phagocytosis, depending on the nature of the antigen. ${ }^{[8,37,39]}$ Receptors in DCs often require co-receptors to form complexes and ligand recognition depends on co-ligands and transporters. Thus, the presence of fluorophores on the particle surface could have modified the particle presentation to the cell receptors, inducing changes in the receptor function and particokinetic alterations. ${ }^{[40]}$ MDDCs internalized the hetero-functional encoded Au-NPs to a greater extent than homo-functional particles (Figure 3a), which can be attributed to the second layer polymer shielding of the first layer fluorophore conjugated polymer (Figure 3b); however these NPs displayed a significantly lower uptake by MDDCs compared to the unlabeled NPs. Despite the shielding effect of the second polymer layer, the difference can most probably still be attributed to the presence of the fluorophore. Polymer coatings are not rigid shells but flexible layers on the surface of NPs, which could result in the presentation of some dye molecules on the particle surface. This is a well-known phenomenon for soft polymer particles. For example, ThomannHarwood et al. ${ }^{[40]}$ showed that the cargo of chitosan particles, i.e. non-solid vaccine carriers, can influence uptake by MDDCs, because these flexible carriers allow parts of a cargo molecule to be exposed on the particle surface. Figure 3c illustrates the impact of the surface functional group, irrespective of the used polymer type. For the three particle series, we show that the presence of amine functional groups in the polymer backbone induced a higher Au-NP uptake than particles coated with polymers containing carboxylic functional groups (Figure 3c) which is in agreement with previously reported studies. ${ }^{[13,15]}$ The hetero-NH2-PVA particles showed the highest cellular uptake in the hetero-functionalized particle series (Figure 3a) which can be confirmed by previous studies with NH2-PVA coated nanoparticles. ${ }^{[15]}$ The lower uptake of carboxyl-functional hetero-encoded particles, 3-fold for $\mathrm{COOH}$ PVA and 9-fold for COOH-(PVA+PEG) and $\mathrm{COOH}-\mathrm{PEG}$, could be explained by their negative charge (Table 1), since it has been shown that negatively charged particles allow for poor interaction with the negative cellular membrane. ${ }^{[13]}$

However, the uptake of the positively charged hetero-encoded particles cannot be explained by the surface charge alone since only for the hetero-NH2PVA Au-NPs a high intracellular particle number could be observed compared to e.g., NH2-(PVA+PEG) particles (Figure 3a). This behavior could be attributed to the PEG polymer. It has been repeatedly shown that PEG coatings can achieve surface "camouflaging" of nanoparticles resulting in prolonged circulation times by avoiding cellular uptake. ${ }^{[41]}$ In general, the presence of a PVA layer resulted in higher cell uptake compared to PEGylated particles (Figure 3d), demonstrating that the physicochemical properties of polymer such as hydrophilicity ${ }^{[42,43]}$ and protein adhesion ${ }^{[4,45]}$ have an important role on MDDC uptake. This result can be transferred to the polymer mixture (PEG and PVA) coated particles where the presence of PVA in the two-component shell displayed a positive effect, i.e, stronger internalization compared to the PEG shell.

ICP-OES data was supplemented by flow cytometry (FACS) to evaluate frequencies of cells that take up encoded particles. With the exception of homo- and hetero NH2-PVA and NH2-(PVA+PEG) Au-NPs, for which we observed high frequencies of positive cells $(71 \%$ and $64 \%)$, FACS analysis revealed comparable uptake for other particles (Figure 4). In addition to ICP-OES findings shown in Figure 3a, this data suggests that not only the relative quantity of NP taken up by cells, but also the relative frequencies of cells involved in NP uptake depends on chemical NP modification. In particular high delta in uptake frequency between 


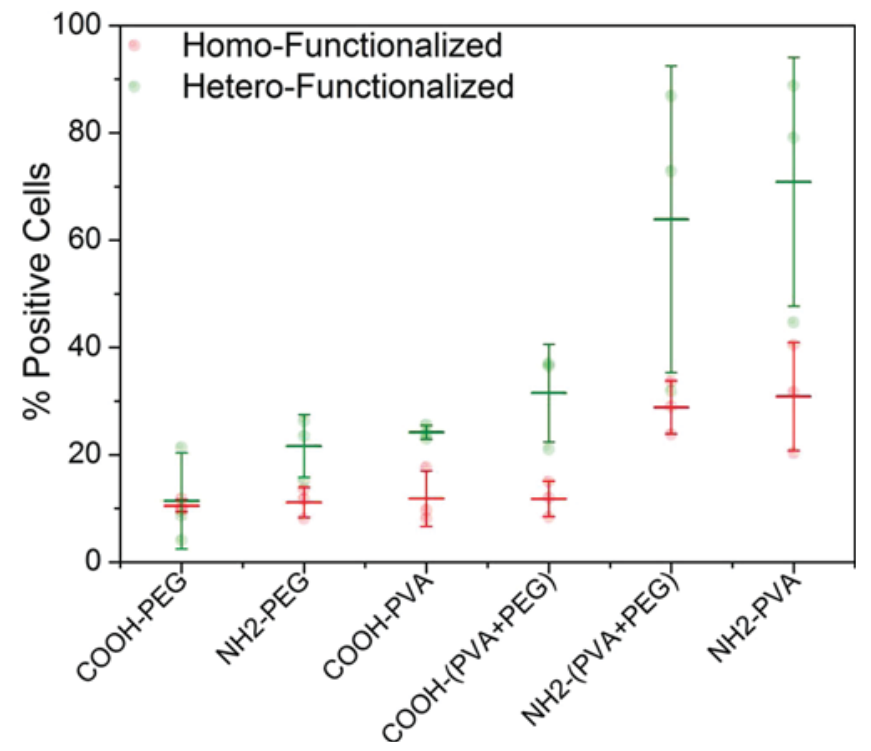

Figure 4. FACS analysis of MDDCs treated with homo-functionalized Au-NPs (red) and hetero-functionalized Au-NPs (green) at a concentration of $20 \mu \mathrm{gmL}^{-1}$ for $15 \mathrm{~h}$. Samples were measured in triplicate (light red, green and blue circles). The horizontal lines indicate the mean.

homo- and hetero-functionalized Au-NPs was observed for the NH2-(PVA+PEG) and NH2-PVA coated NPs, with the hetero-functionalized NPs showing a much higher frequency of positive cells than the corresponding homo-functionalized NPs. Regarding above comparison it is important to note that the number of fluorophores were identical for the homo-and the hetero-encoded NPs and that uptake frequency was therefore compared between NPs showing identical fluorophore density (i.e., 3410 fluorophores/NP for NH2-PVA encoded Au-NPs and 1050 fluorophores/NP for $\mathrm{NH} 2-(\mathrm{PVA}+\mathrm{PEG})$ encoded Au-NPs). However, caution is required when comparing uptake of particles showing large differences in fluorophore content: Measured frequencies of positive cells may vary since a smaller quantity of NPs with high fluorophore grafting density is required to generate a positive signal, as compared to NPs with comparatively lower fluorophore content.

Figure 5 shows the comparison between the 2 methods ICP-OES and FACS as a function of the relative fluorescence intensity. Thus, a better qualitative link was obtained since the variation of the number of dyes/particles was eliminated. This is of course only a rough estimate and more precise and detailed investigations will be carried out in the future.

Further insight into the cellular internalization of the fluorescence-encoded Au-NPs was gained by LSM. Intracellular fluorescent particles are shown in reconstructed 3D illustrations of single MDDC in Figure 6. As expected from the ICPOES and FACS data, we did not detect PEGylated particles by LSM (Figure 6i, 6j, 6k and 6l). For the encoded particles functionalized with PVA and the mixture of both polymers, we observed significant differences between amino- and carboxylic-functional polymers. Weak signals of homo- and hetero-COOH-PVA and $\mathrm{COOH}-(\mathrm{PVA}+\mathrm{PEG})$ encoded $\mathrm{Au}-\mathrm{NPs}$ were detected in few cells, (Figure $6 \mathrm{c}$ and $6 \mathrm{~d}$ for $\mathrm{COOH}-$ PVA and $6 \mathrm{~g}$ and $6 \mathrm{~h}$ for $\mathrm{COOH}-(\mathrm{PVA}+\mathrm{PEG})$ ). In contrast, the hetero-NH2-PVA and NH2-(PVA+PEG) exhibited the strongest fluorescence signal (Figure $6 \mathrm{~b}$ for NH2-PVA and $6 f$ for $\mathrm{NH} 2-(\mathrm{PVA}+\mathrm{PEG})$ ), and fewer signals were detected by their analogues homo-functional particles (Figure 6a for NH2-PVA and 6e for NH2-(PVA+PEG)).

\section{Conclusion}

Fluorescently encoded and well-defined Au-NPs are an important tool to study nanoparticle interaction with single cells in vitro as well as in vivo using standard fluorescence based methods such as FACS or LSM. Therefore we have designed a well-defined and thoroughly characterized library of fluorescence-encoded Au-NPs using two biocompatible polymers and their amine- or carboxy-functional derivatives. Since some of the commonly used analytical methods such as FACS or LSM rely on the fluorescent labeling of

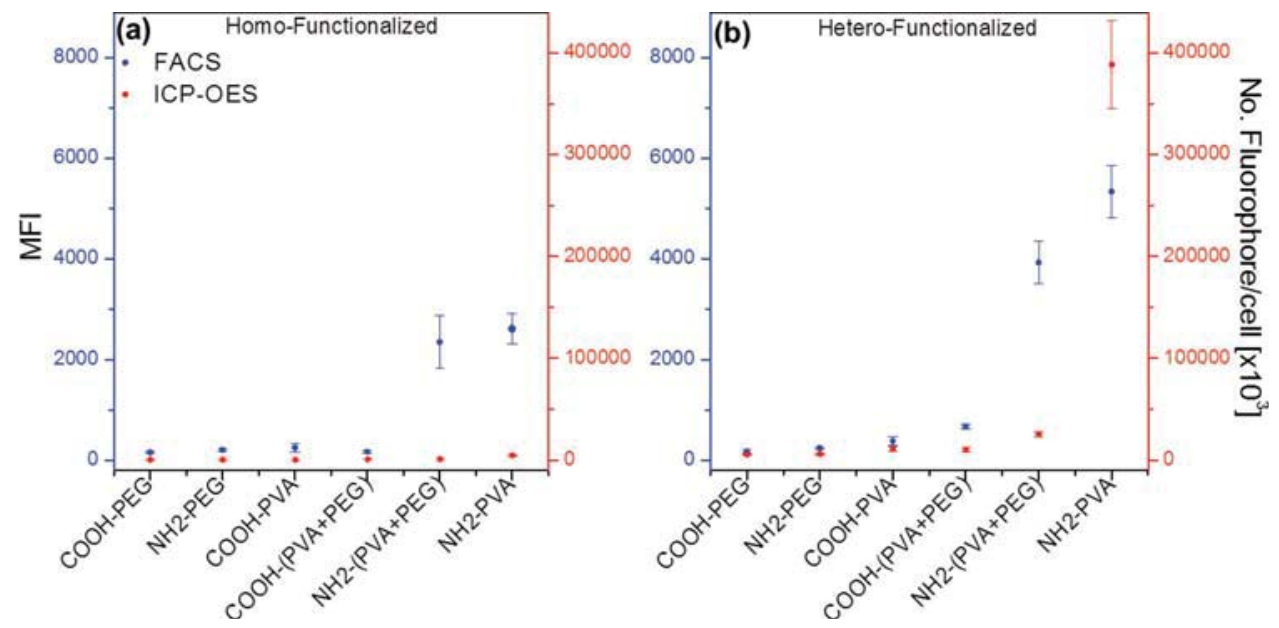

Figure 5. Uptake by MDDCs as a function of surface modification and detection techniques, FACS (blue) and ICP-OES (red): Variation of homofunctionalized Au-NPs (a) and hetero-functionalized Au-NPs (b). Samples were measured in triplicate (error bars $=$ mean \pm SD). 
(a)
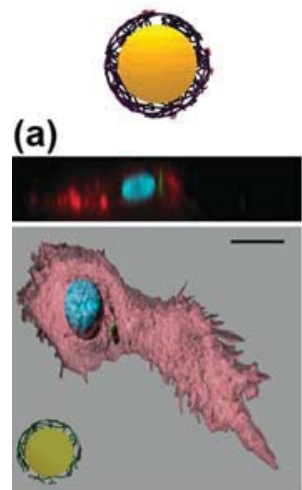

(e)

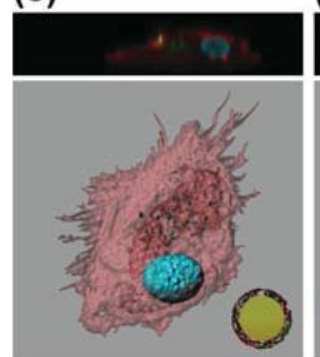

(i)

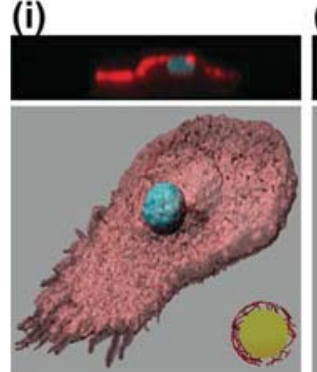

(b)
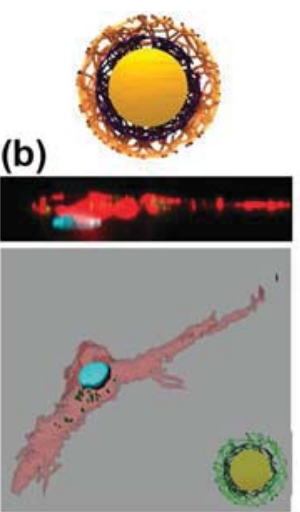

(f)

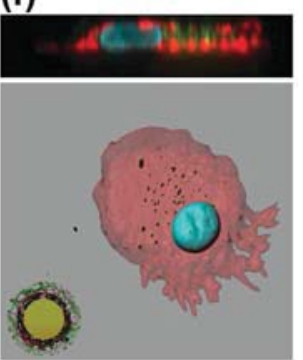

(j)

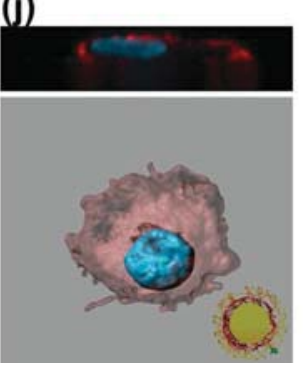

(c)
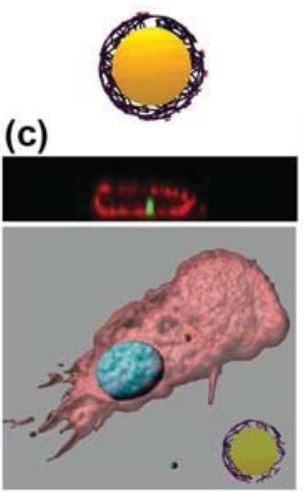

(g)

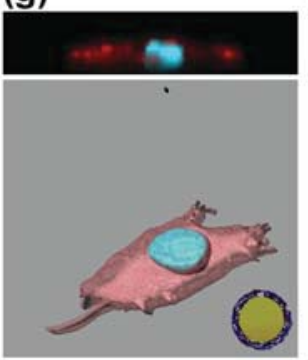

(k)

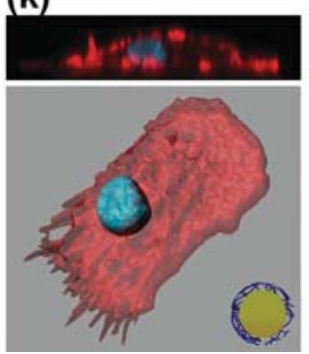

(d)
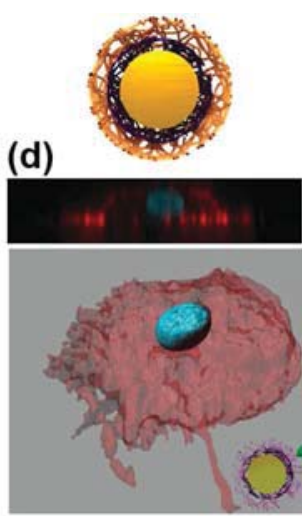

(h)

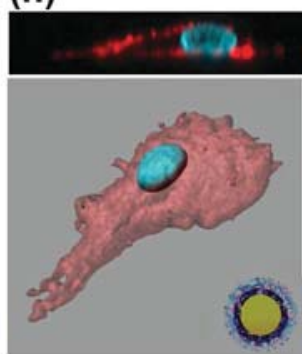

(I)

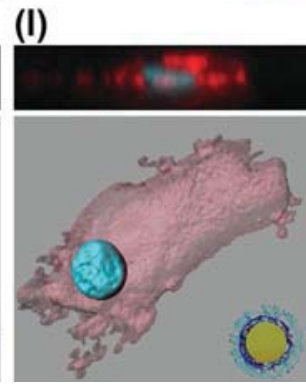

Figure 6. Visualization of fluorescence encoded Au-NPs with laser scanning microscopy. Internalization of homo-functional (a) NH2-PVA, (c) COOH-PVA, (e) NH2-(PVA+PEG), (g) COOH-(PVA+PEG), (i) NH2-PEG and (k) COOH-PEG Au-NPs; and hetero-functional (b) NH2-PVA, (d) COOH-PVA, (f) $\mathrm{NH} 2-(\mathrm{PVA}+\mathrm{PEG}),(\mathrm{h}) \mathrm{COOH}-(\mathrm{PVA}+\mathrm{PEG})$, (j) NH2-PEG and (l) COOH-PEG Au-NPs in MDDCs shown in a 3D reconstruction: Red: F-Actin, Blue: Nucleus, Green: ATT0590-Au-NPs. Scale bar: $7 \mu \mathrm{m}$.

nanoparticles, we have studied the impact of the surface bound dye molecules on cellular uptake. We have shown that the cellular uptake of unlabeled particles that varied significantly with the surface bound polymer was significantly reduced upon addition of the dye. Even more important, all standard homo-functional encoded particles resulted in a very similar low cellular uptake by MDDCs irrespective of their polymer coating and inherent surface charge.

We were able to reduce this masking effect by applying a second polymer layer and we could show that the fluorophore shielding in the hetero-functional encoded particles resulted again in a higher and surface-coating dependent cellular uptake. The impact of the polymer type and surface charge was clearly observable. In contrast, uptake of unlabeled and hetero-functional Au-NPs was modulated by the polymer shell, i. e. with a generally higher uptake of particles containing PVA and particles bearing amine-functional groups on the surface. Thus, the fluorophore, or any structural changes, may define the characteristics of MDDCs uptake and processing. Endocytic capacity in dendritic cells strongly depends on the state of maturation with native cells showing higher rates of internalization than activated cells. Furthermore, endocytosis is complex and includes many different pathways such as phagocytosis, macropinocytosis, clathrinmediated endocytosis, caveolae-mediated endocytosis, among others and its analysis has to be thoroughly optimized for each different cell type. ${ }^{[46]}$ Further studies are required in order to reveal specific endocytic pathways involved in uptake of Au-NPs by dendritic cell.

Here, we also show the importance of applying different analytical methods such as ICP-OES and FACS to quantify the cell uptake of Au-NPs. Both methods yield information about the nanoparticle-cell complex and cannot explicitly distinguish between internalized and membrane attached nanoparticles. Consequently, LSM is absolutely essential to prove the presence of intracellular particles. In addition, ICP-OES and FACS offer different and complementary information about cellular uptake. ICP-OES quantifies the Au content, while FACS measures the number of cells that generate a positive relative fluorescence response. As a consequence, the correlation of both techniques is not evident; therefore future studies should focus on this in much more detail. Our 
results agree well with previous findings and strongly suggest that the structural composition of the NP coating layer, including surface charge and molecular structure, plays an important role for the cellular internalization. Further studies are ongoing to investigate the possible immune-modulatory function of the different encoded Au-NPs in immune cells, such as dendritic cells, in vitro as well as in vivo.

\section{Experimental Section}

Synthesis of ATT0590-Conjugated Vinylalcohol-Vinylamine Copolymer (PVA-NH2) and ATTO590-Conjugated Thiolated PEG Amine: Carbodiimide chemistry was used to conjugate the primary amines of the thiolated PEG (NH2-PEG-SH, Mw 5000, Creative PEGWorks, Winston-Salem, CN, USA) and PVA-NH2 $\left(M_{w} 80000-140000\right.$, Erkol S. A. Spain) with the activated carboxyl groups of the ATT0590 NHS ester (Sigma-Aldrich, Buchs, Switzerland), forming an amide functional group. ${ }^{[47]}$ Briefly, $25 \mathrm{mg}$ of polymer, $100 \mu \mathrm{L}$ of 1-ethyl-3-(3dimethylaminopropyl)-carbodiimide (EDAC; Sigma-Aldrich, Buchs, Switzerland) conjugation buffer (2\% (w/v) EDAC in PBS, $\mathrm{pH} 7$ ) and $60 \mu \mathrm{L}$ of ATTO590 solution ( $2 \mathrm{mg} / \mathrm{mL}$ in DMF) were mixed in a shaker overnight at room temperature. The conjugated polymer was purified by gel filtration chromatography using PD-10 desalting column (Sephadex G-25 medium, exclusion limit $M_{r}=5000$, GE Healthcare Life Sciences) and $0.01 \mathrm{mM}$ PBS buffer as eluent. Then, the purified conjugated polymer was lyophilized for 3 days and stored at $-20^{\circ} \mathrm{C}$. Synthesis of ATT0590-conjugated carboxylic-functional PVA (PVA-COOH). Carbodiimide chemistry was used to conjugate the PVA hydroxide group (carboxyl-modified PVA, Kuraray Specialties Europe $\mathrm{GmbH}$, Hattersheim am Main, Germany) with the activated carboxyl groups of the ATT0590 NHS ester, forming a ester functional group. ${ }^{[47]}$ Briefly, $30 \mathrm{mg}$ of polymer and $200 \mu \mathrm{L}$ EDAC conjugation buffer were mixed in a shaker for $15 \mathrm{~min}$ at room temperature. Then, ATT0590 (60 $\mu \mathrm{L} ; 2 \mathrm{mg} / \mathrm{mL})$ was slowly added to the reaction mixture and immediately the $\mathrm{pH}$ was changed to 5.0 using $\mathrm{HCl}$ allowing the reaction to continue overnight with stirring at room temperature. The conjugated polymer was purified by gel filtration chromatography using PD-10 desalting column and $0.01 \mathrm{mM}$ PBS buffer as eluent. Then, the purified conjugated polymer was lyophilized for 3 days and stored at $-20^{\circ} \mathrm{C}$.

Synthesis of AF594-Conjugated Thiolated PEG Carboxylic Acid: Carbodiimide chemistry was used to conjugate the hydrazine group of AF 594 (Life Technology Europe B. V., Zug, Switzerland) with the carboxyl groups of PEG (COOH-PEG-SH, Mw 5000, Creative PEGWorks, Winston-Salem, CN, USA) forming an amide functional group. ${ }^{[47]}$ Briefly, $35 \mathrm{mg}$ of polymer and $100 \mu \mathrm{L}$ EDAC conjugation buffer ( $2 \%(\mathrm{w} / \mathrm{v})$ EDAC, $3 \%(\mathrm{w} / \mathrm{v}) \mathrm{N}$-hydroxysuccinimide in PBS, $\mathrm{pH}$ 7) were mixed in a shaker for $15 \mathrm{~min}$ at room temperature. Then, $120 \mu \mathrm{L}$ of AF $590(1 \mathrm{mg} / \mathrm{mL}$, DMSO) was added to the activatedPEG-COOH solution and allowed to react overnight with stirring at room temperature. The conjugated polymer was then purified by gel filtration chromatography using $0.01 \mathrm{mM}$ PBS buffer and lyophilized for 3 days. The sample was stored to $-20^{\circ} \mathrm{C}$.

Synthesis of Fluorescence Encoded (Homo- and Hetero) and Unlabeled Homo-Functionalized Au-NPs: Citrate-stabilized $\mathrm{Au}$ nanospheres $(15 \mathrm{~nm}$ in diameter, $[\mathrm{Au}]=0.5 \mathrm{mM}$ ) were synthesized as reported by Enustun and Turkevich. ${ }^{[48]}$ In order to produce the homo-functional encoded particles, PBS $1 \mathrm{x}$ solutions containing an adequate amount of dye-conjugated polymer were prepared. The polymer coating of the Au-NPs was performed to yield 15 molecules $/ \mathrm{nm}^{2}$ for the PEG containing samples, a [Au/ PVA] ratio of 15 for PVA samples, and a [PEG/PVA] ratio of 1 for the mixed samples. Then, the respective polymer solution, which was previously treated by ultrasound for $15 \mathrm{~min}$, was added drop wise under shaking to $10 \mathrm{~mL}$ of synthesized Au-NPs. The mixture was allowed to react at RT for $24 \mathrm{~h}$. Conjugated polymer coated particles were centrifuged (10000 g, $1 \mathrm{~h}$ ) twice to remove excess polymer and re-dispersed in $5 \mathrm{~mL}$ of PBS $1 \mathrm{x}$. For the hetero-functional encoded particles, a second coating with unlabeled polymers was applied using the same conditions. All samples were centrifuged at $10000 \mathrm{~g}$ for $1 \mathrm{~h}$ and re-dispersed in $5 \mathrm{~mL}$ of PBS $1 \mathrm{x}$.

The unlabeled homo-functionalized Au-NPs were synthesized as described above using just the polymers without any fluorophores.

Characterization Methods: Optical characterization was carried out by UV-Vis spectroscopy on a Jasco V-670 spectrophotometer (Jasco Europe S. R. L., Milano, Italy). The UV-Vis spectra were acquired in water, PBS $1 \mathrm{x}$ and RPMI 164 cell culture medium supplemented with $10 \%$ fetal calf serum (FCS to assess the colloidal stability. Emission measurements were recorded using a UV-Vis fluorescence spectrometer (Photon Technology International, Birmingham, UK). Optimization was carried out by performing both excitation and emission scans. The efficiency of the coating steps was monitored through zeta potential measurements (Brookhaven Instruments Corporation, Hotsville, NY, USA). The hydrodynamic radius was measured by dynamic light scattering (DLS) at $80^{\circ}, 90^{\circ}$ and $100^{\circ}$ (LS Instruments A. G., Fribourg, Switzerland).

Cell Culture: Human monocytes were isolated from buffy coat, obtained from healthy volunteers (Blood Donation Service, Bern University Hospital, Bern, Switzerland) by gradient centrifugation (Lymphoprep, Axis Shield, Oslo, Norway) and magnetic separation as described in the MACS Monocyte Isolation Kit Manual (MACS Miltenyi Biotec, Bergisch Gladbach, Germany, Order no. 130-050201). Monocytes were seeded into 12 well plates $\left(2 \mathrm{~mL} /\right.$ well, $10^{6}$ cells $\mathrm{mL}^{-1}$ ) cultured in RPMI 1640 medium (10\% FCS, 1\% L-Glu, 1\% Pen-Strep). The cells were incubated at $37^{\circ} \mathrm{C}, 5 \% \mathrm{CO}_{2}$ for 6 days for differentiation into dendritic cells by additional supplementation of $34 \mathrm{ng} \mathrm{mL}-1$ IL-4 (R\&D Systems Europe Ltd., Abingdon, UK) and $50 \mathrm{ng} \mathrm{mL} \mathrm{L}^{-1} \mathrm{GM}$-CSF (R\&D Systems Europe Ltd., Abingdon, UK).

Nanoparticle Exposure: Upon maturation on day 6, the cells were exposed to $20 \mu \mathrm{gmL}^{-1}$ Au-NPs. After exposure for $15 \mathrm{~h}$, uptake into cells was measured by Flow Cytometry (FACS), Laser Scanning Microscopy (LSM) and Inductively Coupled Plasma Atomic Emission Spectroscopy (ICP-OES). Each experiment was repeated at least three times with cells from different donors.

Cytotoxicity: The ability for unlabeled, homo-functionalized and hetero-functionalized Au-NPs to cause cytotoxicity after $15 \mathrm{~h}$ suspension exposure was determined via the Lactate dehydrogenase (LDH) assay. After exposure of the human MDDCs to Au-NPs the cell supernatants were collected and stored at $4{ }^{\circ} \mathrm{C}$. The experiment was performed according to the manufacturer's specifications (Cytotoxicity Detection Kit, Cat. No.: 22644793 001, Roche Diagnostics, Mannheim, Germany). Supernatants were measured at $490 \mathrm{~nm}$ (with a reference at $630 \mathrm{~nm}$ ) using a multi-plate spectrometer (Benchmark Plus, Bio-Rad, Switzerland). Untreated cells were used as negative control and Triton X-100 0.2 wt\% was used as the positive control at a concentration of $1 \mathrm{wt} \%$ in PBS. Total 
extracellular LDH was then calculated according to the manufacture's manual. In order to account for the interference of the Au-NPs with the colorimetric LDH assay the values of the particles in buffer were subtracted from the values obtained for the cells with incubated nanoparticles.

In addition to the LDH assay we also performed the Trypan Blue exclusion assay. Briefly, exposed human MDDCs were cultured and a cell count was performed using a 1:1 dilution of Trypan Blue (Sigma Aldrich, Buchs, Switzerland) in cell culture media. After three mins at RT, cells $(10 \mu \mathrm{L})$ were counted for each exposure using a haemocytometer. Percentage viability was determined from the positive control (frozen cell cultures at $-80^{\circ} \mathrm{C}$ for $30 \mathrm{~min}$ ). The assays were carried out in triplicate.

Flow Cytometry: Cells were transferred to FACS tubes (1 mL/tube, $10^{6}$ cells $\left.\mathrm{mL}^{-1}\right)$. Cells were kept on ice and washed with FACS buffer ( $1 \% \mathrm{BSA}, 0.1 \% \mathrm{NaN}_{3}$ in PBS), centrifuged ( $500 \mathrm{~g}$ at $4{ }^{\circ} \mathrm{C}$ for $8 \mathrm{~min}$ ) and re-suspended in $400 \mu \mathrm{L}$ FACS buffer. Samples were examined in the LSR Fortessa (BD Biosciences, Allschwill, Switzerland) by using a PE-TEXAS Red channel for the detection of the particles.

ICP-OES: After particle exposure, cells were collected in $15 \mathrm{~mL}$ tubes. They were centrifuged twice $\left(500 \mathrm{~g}\right.$ at $4^{\circ} \mathrm{C}$ for $8 \mathrm{~min}$ ) in order to prevent endo- and exocytosis and remove non-internalized nanoparticles and debris. Supernatants were discarded and cells were digested in $0.5 \mathrm{~mL}$ Aqua Regia $\left(\mathrm{HNO}_{3}: \mathrm{HCl} ; 1: 3\right)$. After $24 \mathrm{~h}$ of digestion, samples were diluted in milli-Q water up to $5 \mathrm{~mL}$. Samples were measured by IPC-OES (Optima 7000DV, Perkin Elmer, Schwerzenbach, Switzerland). Measurements were performed at a wavelength of $242.795 \mathrm{~nm}$ (Limit of detection $(\mathrm{LOD})=16 \mu \mathrm{g} \mathrm{L^{-1 }}$ and limit of quantification ( $\mathrm{LOQ})=23 \mu \mathrm{g} \mathrm{L}^{-1}$ ), at an axial plasma view. The plasma flow was $15 \mathrm{~mL} \mathrm{~min}{ }^{-1}$ and the sample flow rate $1.5 \mathrm{~mL}$ $\mathrm{min}^{-1}$. A washing-step was performed between each measurement and each sample was measured three times.

Immunofluorescence and LSM: Cells were transferred to 4-well culture slides (Cat. No.: 354114, BD Biosciences, Allschwill, Switzerland) and exposed to $20 \mu \mathrm{g} \mathrm{mL}^{-1}$ of Au-NPs for $15 \mathrm{~h}$. After exposure, cells were fixed with $3 \%$ PFA in PBS, permeabilized with $0.2 \%$ Triton-X in PBS and stained with Phalloidin-Alexa488 (Cat. No. A12379, Life-Technologies, Carlsbad, CA, USA) and DAPI (Cat. No. D136, Life Technologies) for F-action and nuclear staining, respectively. Samples were washed, embedded in Glycergel mounting medium (Cat. No. c056330, DAKO, Santa Clara, CA, USA), covered with a coverslip and examined under a ZEISS LSM710 Microscope (Lasers: DPSS $561 \mathrm{~nm}$ for ATTO590 (Au-NPs), HeNe $488 \mathrm{~nm}$ for Alexa Fluor $488 \mathrm{~nm}$ and a UV diode at $405 \mathrm{~nm}$ for DAPI) with an 63× (NA 1.40) objective lens. Visualization and 3D modeling was performed with IMARIS software (Bitplane AG, Zurich, Switzerland).

\section{Supporting Information}

Supporting Information is available

\section{Acknowledgements}

This work was supported by the Swiss National Foundation (PPOOP2-123373/1), the NRP64 program of the SNF, and the
Adolphe Merkle Foundation. The authors acknowledge Dr. Miguel Spuch Calvar for the design of Figure 1, Dr. Dimitri Vanhecke and Dr. Martin Clift for the support with FACS and laser scanning confocal microscope.

[1] H. Fenniri, R. Alvarez-Puebla, Nat. Chem. Biol. 2007, 3, 247-249.

[2] M. Y. Han, X. H. Gao, J. Z. Su, S. Nie, Nat. Biotechnol. 2001, 19, 631-635.

[3] F. Amin, D. A. Yushchenko, J. M. Montenegro, W. J. Parak, ChemPhysChem 2012, 13, 1030-1035.

[4] T. Biver, N. Eltugral, A. Pucci, G. Ruggeri, A. Schena, F. Secco, M. Venturini, Dalton Ttrans. 2011, 40, 4190-4199.

[5] D. N. Heo, D. H. Yang, H. J. Moon, J. B. Lee, M. S. Bae, S. C. Lee, W. J. Lee, I. C. Sun, I. K. Kwon, Biomaterials 2012, 33, 856-866.

[6] A. D. Lehmann, W. J. Parak, F. Zhang, Z. Ali, C. Rocker, G. U. Nienhaus, P. Gehr, B. Rothen-Rutishauser, Small 2010, 6, 753-762.

[7] K. J. Zhou, H. M. Liu, S. R. Zhang, X. N. Huang, Y. G. Wang, G. Huang, B. D. Sumer, J. M. Gao, J. Am. Chem. Soc. 2012, 134, 7803-7811.

[8] F. Blank, P. Gerber, B. Rothen-Rutishauser, U. Sakulkhu, J. Salaklang, K. De Peyer, P. Gehr, L. P. Nicod, H. Hofmann, T. Geiser, A. Petri-Fink, C. Von Garnier, Nanotoxicology 2011, 5, 606-621.

[9] X. Michalet, F. F. Pinaud, L. A. Bentolila, J. M. Tsay, S. Doose, J. J. Li, G. Sundaresan, A. M. Wu, S. S. Gambhir, S. Weiss, Science 2005, 307, 538-544.

[10] R. Weissleder, Nat. Rev. Cancer 2002, 2, 11-18.

[11] J. Zhao, A. O. Pinchuk, J. M. Mcmahon, S. Z. Li, L. K. Ausman, A. L. Atkinson, G. C. Schatz, Acc. Chem. Res. 2008, 41, 1710-1720.

[12] E. C. Dreaden, A. M. Alkilany, X. H. Huang, C. J. Murphy, M. A. El-Sayed, Chem. Soc. Rev. 2012, 41, 2740-2779.

[13] D. Huhn, K. Kantner, C. Geidel, S. Brandholt, I. De Cock, S. J. H. Soenen, P. R. Gil, J. M. Montenegro, K. Braeckmans, K. Mullen, G. U. Nienhaus, M. Klapper, W. J. Parak, ACS Nano 2013, 7, 3253-3263.

[14] R. A. Sperling, W. J. Parak, Ther. Innov. Regul. Sci. 2013, 47, 1333-1383.

[15] V. Hirsch, C. Kinnear, M. Moniatte, B. Rothen-Rutishauser, M. J. D. Clift, A. Fink, Nanoscale 2013, 5, 3723-3732.

[16] D. Shenoy, W. Fu, J. Li, C. Crasto, G. Jones, C. DiMarzio, S. Sridhar, M. Amiji, Int. J. Nanomed. 2006, 1, 51-57.

[17] A. P. Alivisatos, K. P. Johnsson, X. G. Peng, T. E. Wilson, C. J. Loweth, M. P. Bruchez, P. G. Schultz, Nature 1996, 382, 609-611.

[18] R. Lévy, U. Shaheen, Y. Cesbron, V. Sée, Nano Rev. 2010, 1, 4889.

[19] P. Rivera-Gil, D. Jimenez de Aberasturi, V. Wulf, B. Pelaz, P. del Pino, Y. Zhao, J. M. De La Fuente, I. Ruiz de Larramendi, T. Rojo, X.-J. Liang, W. J. Parak, Acc. Chem. Res. 2013, 46, 743-749.

[20] B. D. Chithrani, W. C. W. Chan, Nano Lett. 2007, 7, 1542-1550.

[21] D. Napierska, L. C. J. Thomassen, V. Rabolli, D. Lison, L. Gonzalez, M. Kirsch-Volders, J. A. Martens, P. H. Hoet, Small 2009, 5, 846-853.

[22] E. Casals, T. Pfaller, A. Duschl, G. J. Oostingh, V. F. Puntes, Small 2011, 24, 3479-3486.

[23] E. Hutter, S. Boridy, S. Labrecque, M. Lalancette-Hebert, J. Kriz, F. M. Winnik, D. Maysinger, ACS Nano 2010, 4, 2595-2606.

[24] P. Kolhar, A. C. Anselmo, V. Gupta, K. Pant, B. Prabhakarpandian, E. Ruoslahti, S. Mitragotri, Proc. Natl. Acad. Sci. U S A 2013, 26, 10753-10758.

[25] Y. C. Wang, K. C. L. Black, H. Luehmann, W. Y. Li, Y. Zhang, X. Cai, D. H. Wan, S. Y. Liu, M. Li, P. Kim, Z. Y. Li, L. H. V. Wang, Y. J. Liu, Y. A. Xia, ACS Nano 2013, 7, 2068-2077. 
[26] J. C. Love, L. A. Estroff, J. K. Kriebel, R. G. Nuzzo, G. M. Whitesides, Chem. Rev. 2005, 105, 1103-1169.

[27] Y. Q. Tu, P. Wu, H. Zhang, C. X. Cai, Chem. Commun. 2012, 48, 10718-10720.

[28] S. Chowdhury, Z. Wu, A. Jaquins-Gerstl, S. Lui, A. Dembska, B. A. Armitage, R. Jin, L. A. Peteanu, J. Phys. Chem. C 2011, 115, 20105-20112.

[29] T. L. Jennings, M. P. Singh, G. F. Strouse, J. Am. Chem. Soc. 2006, $128,5462-5467$.

[30] F. Zhang, Z. Ali, F. Amin, A. Feltz, M. Oheim, W. J. Parak, Chem. Phys. Chem. 2010, 11, 730-735.

[31] J. Manson, D. Kumar, B. J. Meenan, D. Dixon, Gold Bull. 2011, 44, 99-105.

[32] A. R. Khare, N. A. Peppas, Biomaterials 1995, 16, 559-567.

[33] R. Skouri, F. Schoesseler, J. P. Munch, S. J. Candau, Macromolecules 1995, 28, 197-210.

[34] K. Abe, S. J. Senoh, Polym. Sci. A 1986, 24, 3461-3474.

[35] J. Cho, F. Caruso, Macromolecules 2003, 36, 2845-2851.

[36] Y. Zhang, H. Pan, P. Zhang, N. Gao, Y. Lin, Z. Luo, P. Li, C. Wang, L. Liu, D. Pang, L. Cai, y. mA, Nanoscale 2013, 5, 5919-5929.

[37] S. E. A. Gratton, P. A. Ropp, P. D. Pohlhaus, J. C. Luft, V. J. Madded, M. E. Napier, J. M. DeSimone, Proc. Natl. Acad. Sci. USA 2008, 105, 11613-11618.

[38] A. Albanese, W. C. W. Chan, ACS Nano 2011, 5, 5478-5489.
[39] P. G. Holt, P. A. Stumbles, J. Aerosol. Med. 2000, 13, 361-367.

[40] L. J. Thomann-Harwood, P. Kaeuper, N. Rossi, P. Milona, B. Hermann, K. C. McCullough, J.Controlled Release 2013, 166, 95-105.

[41] C. Brandenberger, C. Muhlfeld, Z. Ali, A. G. Lenz, O. Schmid, W. J. Parak, P. Gehr, B. Rothen-Rutishauser, Small 2010, 6, 1669-1678.

[42] M. H. Priya, L. R. Pratt, M. E. Paulaitis, Langmuir 2011, 27, 13713-13718.

[43] S. K. Sahoo, J. Panyam, S. Prabha, V. Labhasetwar, J.Controlled Release 2002, 82, 105-114.

[44] O. Inui, Y. Teramura, H. Iwata, ACS Appl. Mater. Interface 2010, 2, 1514-1520.

[45] T. A. Larson, P. R. Joshi, K. Sokolov, ACS Nano 2012, 6, 9182-9190.

[46] B. Rothen-Rutishauser, D. A. Kuhn, Z. Ali, M. Gasser, F. Amin, W. J. Parak, D. Vanhecke, A. Fink, P. Gehr, C. Brandenberger, Nanomedicine 2013, doi:10.2217/nnm.13.24.

[47] G. T. Hermanson, Bioconjugate Techniques 2nd ed., Elsevier Inc., Rochford, USA, 2008.

[48] B. V. Enustun, J. Turkevich, J. Am. Chem. Soc. 1963, 85, 3317-\&. 